\title{
Utilizing in vitro transporter data in IVIVE-PBPK: an overview
}

\author{
Pankajini Mallick \\ College of Pharmacy, Department of Pharmacological and Pharmaceutical Sciences, University of Houston, Houston, TX \\ 77030, USA
}

*Corresponding Author: E-mail: pankajini.mallick@gmail.com; Tel.: 832-842-8343

Received: September 18, 2017; Revised: September 30, 2017; Published: December 24, 2017

\begin{abstract}
In vitro-in vivo extrapolation (IVIVE) integrated in physiologically-based pharmacokinetic (PBPK) models have been increasingly used during drug discovery and development processes to predict human pharmacokinetic (PK) parameters. Drug transporters can influence drug pharmacokinetics and are key aspects contributing to the development of a successful drug. This review provides a snapshot of challenges or shortcomings of in vitro and in vivo techniques for understanding the contribution of drug transporters to a drug's pharmacokinetics. The paper also describes the potential of IVIVE-PBPK models as prospective approaches to predict the role of drug transporters in drug discovery and development.
\end{abstract}

\section{Keywords}

Pgp; Bile acid transporter; Drug transporters; Drug metabolizing enzymes; Drug induced liver injury

\section{Introduction}

The role of drug transporters in drug efficacy and safety has been of much interest; drug transports play key roles in drug absorption, distribution, and excretion [1-3]. In drug discovery and development, drug transporters expressed in the intestine, liver, kidneys, and brain have received considerable attention [4-7]. Many of the drug transporters that have been cloned and characterized belong to two major superfamilies, ATP-binding cassette $(A B C)$ and solute carrier ( $S L C)$. Major efflux transporters of the $A B C$ family include MDR1 (P-glycoprotein, ABCB1), BCRP (ABCG2), and MRP2 (ABCC2), and are localized to barrier tissues in the body, such as the intestine, liver, kidneys, blood-brain barrier, and placenta [8-11]. Absorption of a drug is a key component to achieve good bioavailability, and for oral drugs, the majority of drug absorption occurs in the small intestine where the presence of villi and microvilli greatly increases the surface area for optimal absorption. Thus, drug-transporter interaction in the intestine often contributes to poor absorption, resulting in low bioavailability. Given that oral delivery is the preferred route of administration for drugs, intestinal transporters are potential targets to achieve optimal clinical oral plasma exposure for hydrophilic and polar drugs [12]. Although the significance of drug transporters in absorption, distribution, metabolism, and elimination (ADME) of drugs is well recognized, a knowledge gap exists in terms of change in drug transporter functionality (their amount and activity) in response to disease, and how pathophysiological pathways change transporter expressions [13]. Because of the central role of the liver in drug metabolism, the role of transporters in the liver in drug interactions and drug responses, as well as drug-induced liver injury, has been in focus of late. 
Despite a steady growth in the field of transporter biology, which has bolstered transporter-mediated therapeutics, the physiological functions of many transporters are still largely unknown. These growing trends bring challenges for drug development, as well as for regulatory agencies, with regard to identification of membrane transporters that influence the disposition and safety of drugs, and also optimization of membrane permeability to improve oral absorption of new chemical entities. Thus, utilization of in vitro transporter assays has become a critical tool for assessing a drug's potential in vivo absorption properties and drug-drug interactions [14]. Use of in vitro-in vivo extrapolation (IVIVE) integrated in physiological-based pharmacokinetic (PBPK) models, together known as IVIVE-PBPK models, has gained recent momentum in the pharmaceutical industry. Since variation in protein expression of drug metabolizing enzymes and transporters is known to be a major complicating factor in IVIVE, the accurate quantification of these proteins in complex biological systems is essential for improving IVIVE-based pharmacokinetic predictions [15]. This review focuses on the use and shortcomings of qualitative and quantitative methods used to create drug transporter-based IVIVE-PBPK models in the liver, intestine, and brain.

\section{Pitfalls of in vitro and in vivo systems used in drug transporter studies}

In vitro cell-based systems are used routinely to elucidate passive and active transporter processes acting to influence drug permeability. Different in vitro systems include use of membrane vesicles or transfected cell lines for drug transporter studies; however, there are several pitfalls in estimating transporter kinetics using these conventional approaches [16]. Considering that one drug can be transported by more than one uptake or efflux transporter, a limitation of these systems is the presence of a single transporter of interest. Further, given that there exists an interplay between drug metabolizing enzymes and transporters, cell lines lacking metabolism machinery pose another limitation to their use. In vitro experiments can lead to biased estimation of apparent $-K_{m}$, the biochemical constant in experiments that assumes similar $K_{\mathrm{m}}$ for both apical and basal transport directions [17-20]. Another critical factor whether intracellular concentrations that drive efflux transport are accounted for [21]. Drug concentrations within the donor and acceptor compartments are dynamic; thus, the assumption that unbound drug concentrations on either side of a membrane are in thermodynamic equilibrium $[22,23]$ is not applicable for drugs that are poorly permeable, actively transported, or extensively metabolized.

A host of in vitro assays have been developed to mimic the complexity of biological systems; however, many questions remain regarding the translation of in vitro information to in vivo. Therefore, in vivo systems provide the ultimate determination of the effect of drug transporters on disposition of drugs. Transgenic and mutant animal models have provided an important tool for assessing drug efflux transporter activity. To this end, the efflux transporters P-glycoprotein (P-gp) and multidrug resistanceassociated protein (Mrp1) have been the most frequently investigated in transgenic animals [24-26]. Like in vitro systems, in vivo systems also have limitations. Mice generally have two genes to encode transporter proteins, and silencing one of these genes may not sufficiently suppress the expression of the protein [25]. Moreover, efflux transporters may work through a compensatory mechanism, where absence of one gene might be compensated for by another transporter gene [27]. Furthermore, due to the ubiquitous expression of drug efflux transporters throughout the body, the removal of a gene or genes affects tissues other than the organ of interest. This can lead to undesirable outcomes such as altered pharmacokinetics, lack of viability, or systemic toxicity. To get around these limitations, specific transporters can be modulated with various agents [28]. The advantage of using drug transporter modulators is that these studies are generally performed with wild-type animals, and thus, the effect of a compensatory mechanism 
is not so important. However, due to overlap in substrate specificities, and lack of specificity, drug efflux transporter modulation is not as effective as gene knockout models [28]. Further, if drug transporter modulators are dosed orally, it is often difficult to get an effective dose to the targeted organ; this is likely be due to first pass metabolism. Even with intraperitoneal dosing, the limited effective dose of the modulator could be because of physiochemical properties of the modulator, such as protein binding and/or unfavorable tissue distribution [29]. Importantly, the effects of modulators on drug transporters are transient and concentration dependent; therefore, proper dosing and study time points becomes critical variables in these studies. There exists potential species-specific differences in drug transporter activities and contributions, as exhibited in MDR1 functional studies, in which some compounds showed affinity for MDR1A/B activity in mouse MDR1 cells but not in human cells [30].

Currently available in vitro and in vivo models have a limited capability to quantitatively predict the impact of transporters on intracellular drug concentrations. As PBPK modeling approaches consider physicochemical properties of drugs, in vitro cell-based passive permeability, and cellular uptake/efflux, as well as in vivo animal and human pharmacokinetic data to estimate unbound intracellular drug concentrations in different tissues, this approach provides an effective platform over currently available in vitro and in vivo models. However, these models involve a number of assumptions. Use of in vitro models, such as three-dimensional cultured-hepatocytes that retain the activity of both apical and basolateral hepatic drug transporters, drug metabolizing enzymes, or in vivo multiple gene/tissue-selective knockouts, serves as an improved medium to bolster refinement of PBPK models for better prediction in humans.

\section{IVIVE-PBPK}

PBPK models offer a quantitative mechanistic platform for predicting drug pharmacokinetics. When modeled for pharmacodynamics, these models can be used to predict clinical efficacy and safety with varying drug doses. The IVIVE approach is well established for many drug metabolizing enzymes [31, 32]; however, the integration of drug transporters into PBPK models is lacking, with few positive examples reported [33-36]. IVIVE-PBPK models link in vitro systems to the in vivo system through algorithms and scaling factors [37, 38]. Therefore, for an effective IVIVE-PBPK model to predict the behavior of a drug in vivo, accurate and relevant in vitro data in conjunction with the model system parameters of passive permeability and transporter-mediated flux are necessary.

Static models used alongside PBPK models in drug development are simple and require limited input data; however, due to simplification, these often result in conservative estimates, a lack of ability to capture parallel clearance and elimination pathways, are not suitable for non-linear kinetics, and lack physiology inputs. Although PBPK models require extensive in vitro and in vivo data, they can capture transporter-mediated disposition, describe physiology, and can be applied for special populations such as pediatric patients, in which data is generally very limited.

\section{PBPK for the prediction of transporter-mediated drug induced liver toxicity}

Membrane transporters expressed on hepatocytes and enterocytes play a critical role in homeostasis of endobiotics, e.g., bile acids. Homeostasis of bile acid is maintained through enterohepatic circulation, which allows movement of bile acid molecules from the liver to the small intestine and back to the liver. Bile acid transporter, also known as apical sodium-dependent bile acid transporter, is considered a major determinant of bile acid homeostasis in the body, and is an essential regulator of lipid and cholesterol homeostasis [39-41]. Impaired bile acid export may lead to increased concentrations of liver bile acids, causing hepatocellular apoptosis and/or necrosis. Thus, drugs with the potential to inhibit these 
transporters can disturb the disposition of both co-administered drugs and bile acid, contributing to the development of cholestatic drug-induced liver injury (DILI) $[42,43]$. In vitro studies with isolated membrane vesicles have demonstrated an association between DILI and the inhibitory effects of drugs on bile acid efflux transporters [43-45]. In vitro findings may not translate directly to in vivo hepatotoxicity risk for various reasons, including complexity of bile acid homeostasis, feedback regulation of bile acid synthesis and transport, and dynamic drug/metabolite concentrations in the system [46, 47]. In vivo studies report that preclinical animals are less sensitive to DILI caused by bile acid transporter inhibition, as compared to humans $[48,49]$. Therefore, mechanistic models that integrate physiological information and in vitro experimental data to evaluate DILI mechanisms may be useful to prospectively predict hepatotoxic potential of new drug candidates [50]. The development of a mechanistic model to predict hepatic bile acid transport mediated DILI of troglitazone (TGZ) [51] demonstrated the significant contribution of PBPK modeling to the prediction of hepatotoxicity due to the inhibition of drug transporters, and the potential of a transporter-mediated PBPK model to aid drug development. The mechanistic PBPK model used to study the involvement of bile acid homeostasis in TGZ hepatoxicity incorporated drug/metabolite disposition, bile acid physiology/pathophysiology, and hepatocyte life cycle. Large population variability in bile acid exposure makes prediction of bile acid-mediated hepatotoxicity challenging; hence, the model included population-based analysis to overcome population variability and low incidence of hepatotoxicity. In a rat PBPK model, TGZ was not hepatotoxic; however, in a stimulated human PBPK model, TGZ resulted in a delayed increase in serum alanine aminotransferase (ALT) (marker of liver injury). Thus, the variability added to the parameters describing drug disposition, body weight, and sensitivity of ATP synthesis to hepatic bile acid accumulation, allowed prediction of the incidence of TGZ hepatotoxicity and the species specific bile acid-mediated DILI mechanism.

\section{Intestinal transporters linked to IVIVE-PBPK model for oral absorption}

The bioavailability of an orally administered drug is primarily dependent on the transporters expressed on the apical/basolateral side of enterocytes that influence both the fraction of drug absorbed $[52,53]$ and the fraction escaping gut metabolism. It is difficult to reproduce the complexity of the human gut within a laboratory setting; therefore, efforts are being made to incorporate drug absorption into PBPK models by taking into account several physiological elements of the gastro-intestinal tract, e.g., $\mathrm{pH}$, active transporters, and metabolizing enzymes [54]. To mechanistically develop a PBPK model, the intestine essentially accounts for the region-specific anatomical and physiological differences. Advanced models such as the advanced dissolution, absorption, and metabolism (ADAM) model and the advanced compartmental absorption and transit model (ACAT) are based on adaptations of the original compartmental absorption and transit (CAT) model which accounts for small intestinal transit time (SI), permeability, and radii. Most importantly, these advanced models incorporate gastrointestinal transporter-metabolism interplay [55, 56]. A study by Bruyere and co-workers has highlighted the importance of obtaining regional intestinal transporter expression data for incorporation in PBPK models [57]. This study estimated the scaling factor based on protein estimation of both drug metabolizing enzymes and transporters across the small intestine; these were used for predicting intestinal clearance and for the development of an IVIVE-PBPK model. The results demonstrated that accounting for the MDR1 distributions enhanced PBPK predictions of bioavailability for the development of the compound under study. Likewise, a study by Darwich and coworkers [56], which incorporated regional MDR1 and CYP3A expression into an ADAM model, demonstrated two points: non-uniform regional distribution of MDR1 in the small intestine and colon, and disparity between mRNA and protein expression. This highlights that protein and mRNA expression together with drug parameter data leads to successful capture of drug disposition. The mechanistic PBPK 
absorption model of oxybutynin (OXY) was based on a CAT model that predicts the fraction absorbed based on permeability data in order to predict OXY's oral bioavailability, for two different formulations: immediate-release (IR) and modified-release (MR) [58]. As the primary objective is to predict the fraction absorbed, this multi-compartmental absorption model was a simplified version of a CAT model used to divide the small intestine into three compartments instead of seven, including the duodenum, jejunum, and ileum. The model allowed the drug amount to be modelled, either in solid or dissolved state, for each segment. Assumptions made included: well mixed Gl compartments, no drug degradation in the Gl tract, only the dissolved drug to be absorbed, and absorption to be a non-saturable process. Transfer of mass between adjacent segments was considered to follow first-order kinetics, controlled by a rate constant of gastric emptying. The dissolution rate of OXY was modeled based on spherical particles dissolving over time [55]. Further, OXY's regional intestinal metabolism was modeled through an enterocyte compartment, with the assumptions that the metabolism in the liver and intestine was equal, and there was no binding to enterocytes. The model showed that the fraction absorbed from the formulation was reduced compared to $I R$, despite higher intestinal availability of the formulation. Considering the assumption that the fraction remaining in the liver and intestine is the same, the model prediction supported the hypothesis that higher bioavailability with the OXY formulation is due to increased intestinal availability.

\section{IVIVE-PBPK model for the central nervous system}

Given the high failure rate of drugs developed for central nervous system (CNS) indications [59], early and accurate prediction of drug penetration across the blood-brain barrier (BBB) is vital during drug development. Further, the prediction of brain penetration for non-CNS drugs is important during drug development, in order to avoid unwanted neurotoxicity. Human pharmacokinetic data from the brain is highly restricted; thus, PBPK modeling is of significant value as it can be used to predict the target site concentrations in inter-species and inter-disease situations [60-62]. However, until recently, there has been relatively few published IVIVE-PBPK models to predict BBB permeability, mostly due to limitations in the current in vitro systems used to represent the complex and transporter-rich structure of the in vivo BBB, and due to difficulty in obtaining the appropriate in vitro-in vivo scaling factors [63]. A generic multicompartmental CNS distribution model structure proposed by Yamamoto et al [64], based on the compounds acetaminophen, atenolol, methotrexate, morphine, paliperidone, phenytoin, quinidine, remoxipride, and risperidone, successfully described the pharmacokinetics in plasma and different CNS compartments [64]. Prediction of this multi-compartmental brain PK model matched well with the observed concentration-time profiles, adequately describing the data for all compounds studied. The model structure comprised physiologically relevant brain compartments including brain extracellular fraction (brainECF), brain intracellular fluid compartment (brainICF), compartment of cerebrospinal fluid (CSF) in lateral ventricle (CSFLV), compartment of CSF in third and fourth ventricle (CSFTFV), compartment of CSF in cisterna magna (CSFCM), and CSF subarachnoid space (CSFSAS). Thus, it could successfully describe the PK in plasma and different CNS compartments using microdialysis data. Recently, there has been an increasing focus on translational modelling approaches compared to the traditional practice of fitting model parameters to preclinical in vivo data [65, 66]. IVIVE-PBPK models reported by Fenneteau et al [67] and Ball et al [68] demonstrate promising examples. This review has addressed imperative differences among various PBPK models in use, including details on brain compartmentalization and parameterization. The general approach of using in vivo data to obtain drug specific parameters and physiological parameter estimates by fitting to in vivo data has poor predictivity. Similar to empirical PBPK models, the IVIVE-PBPK model uses a bottom-up approach through IVIVE, with the use of appropriate physiological scaling factors. Fenneteau and colleagues scaled in vitro passive and efflux permeabilities of domperidone to in vivo 
intrinsic permeabilities, and then using literature-based physiological values for in vivo membrane surface area, they further scaled the permeabilities to whole organ permeabilities [67]. The in vitro intrinsic P-gp efflux permeability determined in Caco-2 cells was corrected using the relative fraction of MDR1A/1B messenger RNA expression measured in the brain compared to that in the intestine.

Two methods of IVIVE used for PBPK modelling of morphine and oxycodone describe different strategies for IVIVE of drug transporters at the BBB, which can be used in different phases of drug development [69]. The morphine IVIVE-PBPK model was based on data from in vitro Caco-2 permeability and in vivo total concentrations in brain homogenate. On the other hand, the oxycodone IVIVE-PBPK model used data from complicated experiments generally performed in later stage of drug development, which included in vivo unbound extracelluar fraction (ECF) concentrations obtained from microdialysis and in vitro uptake kinetics in rat brain microvessel endothelial cells. Relative activity factor (RAF) was used to account for the difference between in vitro and in vivo data. Since it is very difficult to obtain human brain homogenate or primary brain endothelial cells from isolated microvessels, determination of drug-specific parameters from in vitro systems of human origin is not always feasible. Brain microdialysis and cerebrospinal fluid (CSF) sampling is another approach to determine drug pharmacokinetics, but due to the invasive nature of these techniques, they are only carried out on diseased patients [70], which introduces significant challenges for the evaluation of model predictions with the human CNS PBPK model. Linking PBPK/PD models to biomarkers or observed in vivo pharmacodynamics are alternative approaches to evaluate human CNS PBPK predictions. However, the success of these approaches depends on selection of appropriate biomarkers and the establishment of a reliable PK/PD relationship. Thus, there is need for more IVIVE-PBPK models to fully explore and evaluate their applicability for the prediction of drug pharmacokinetics in the brain.

\section{IVIVE-PBPK model for transporter mediated drug-drug interaction}

Traditional pharmacokinetic modeling lacks good predictability for complex drug-drug interactions (DDIs) because in most cases, only in vitro and limited clinical data are available in early drug development. As IVIVE-PBPK enables scaling of early in vitro and animal data for human predictions, it can aid DDI risk assessment. The effect of transporter-enzyme interplay on drug bioavailability and hepatic disposition is well recognized [71, 72].There are examples of the involvement of transporter-enzyme interplay in DDIs. Lau et al reported that the inhibitory effect of rifampin on atorvastatin kinetics involves transporter-enzyme interplay [73]. Rifampin is a potent inhibitor of organic anion-transporting polypeptide (OATP) transporters and atorvastatin is primarily metabolized by CYP3A in the liver, via its lactone group. In the study by Lau et al [73], rifampin demonstrated inhibition of atorvastatin uptake into the liver and a change in the ratio of atorvastatin lactone to atorvastatin acid, thereby changing the amount of substrate available to the enzymes in the liver. Grillo et al [74] demonstrated the use of IVIVE-PBPK modeling to predict potential clinically relevant DDI between rivaroxaban and a combination of P-gp and a moderate CYP3A4 inhibitor in renal impaired patients. Thus, bottom-up PBPK models are a powerful tool for the prediction of transporter-mediated drug interactions, and accurate quantitation of protein concentrations in in vitro and in vivo systems will further enhance their accuracy. Given that in vitro systems do not completely reproduce the transport rates and affinities like in vivo, the middle out parameter optimization approach is a necessary step in model verification.

\section{Conclusions}

IVIVE requires information from numerous processes and organs. Each has its advantages and limitations in translating data to the clinic. IVIVE-PBPK modeling is a powerful approach when full kinetic 
characterization of transporters is implemented. Thus, understanding the influence of drug transporters on drug pharmacokinetics can be obtained through a combination of approaches. The future of drug transporter-based IVIVE-PBPK models lies in their potential to contribute to pharmacogenetic studies. Drug transporters and metabolizing enzymes differ significantly based on various factors, including gender, age, race/ethnicity, genetics (polymorphisms), disease state, diet, and lifestyle. The impact of polymorphisms in drug metabolizing enzymes on pharmacokinetic variation is largely studied and known; however, knowledge regarding the impact of polymorphisms in drug transporters is limited. Studies undertaken to date report significant changes in intracellular drug levels due to polymorphisms in drug transporters, which suggests potential alterations in the systemic pharmacokinetics of drugs. Most drug transporters (e.g., P-gp and MRP-1) follow a developmental ontogeny and like most drug metabolizing enzymes, the activities of membrane transporters are immature in neonates. Thus, future research is needed to drive system-dependent scaling factors for predicting transporter contributions of new molecular entities, in different disease states and among special populations, using IVIVE-PBPK modeling approaches.

\section{Conflict of interest: None}

\section{References}

[1] R.P. Oude Elferink, D.K. Meijer, F. Kuipers, P.L. Jansen, A.K. Groen, G.M. Groothuis. Hepatobiliary secretion of organic compounds; molecular mechanisms of membrane transport. Biochim Biophys Acta 1241 (1995) 215-268.

[2] L. Zhang, C.M. Brett, K.M. Giacomini. Role of organic cation transporters in drug absorption and elimination. Annu Rev Pharmacol Toxicol 38 (1998) 431-460.

[3] H. Kusuhara, Y. Sugiyama. Role of transporters in the tissue-selective distribution and elimination of drugs: transporters in the liver, small intestine, brain and kidney. J Control Release 78 (2002) 43-54.

[4] M. Muller, P.L. Jansen. Molecular aspects of hepatobiliary transport. Am J Physiol 272 (1997) G1285-1303.

[5] H. Koepsell. Organic cation transporters in intestine, kidney, liver, and brain. Annu Rev Physio/ 60 (1998) 243-266.

[6] D.K. Meijer, G.J. Hooiveld, A.H. Schinkel, J.E. van Montfoort, M. Haas, D. de Zeeuw, F. Moolenaar, J.W. Smit, P.J. Meier. Transport mechanisms for cationic drugs and proteins in kidney, liver and intestine: implication for drug interactions and cell-specific drug delivery. Nephrol Dial Transplant 14 Suppl 4 (1999) 1-3.

[7] H. Suzuki, Y. Sugiyama. Transporters for bile acids and organic anions. Pharm Biotechnol 12 (1999) 387-439.

[8] D. Lautier, Y. Canitrot, R.G. Deeley, S.P. Cole. Multidrug resistance mediated by the multidrug resistance protein (MRP) gene. Biochem Pharmacol 52 (1996) 967-977.

[9] G.J. Hooiveld, J.E. van Montfoort, D.K. Meijer, M. Muller. Function and regulation of ATP-binding cassette transport proteins involved in hepatobiliary transport. Eur J Pharm Sci 12 (2001) 525-543.

[10] G. Lee, S. Dallas, M. Hong, R. Bendayan. Drug transporters in the central nervous system: brain barriers and brain parenchyma considerations. Pharmacol Rev 53 (2001) 569-596.

[11] N. Mizuno, T. Niwa, Y. Yotsumoto, Y. Sugiyama. Impact of drug transporter studies on drug discovery and development. Pharmacol Rev 55 (2003) 425-461.

[12] M.V. Varma, C.M. Ambler, M. Ullah, C.J. Rotter, H. Sun, J. Litchfield, K.S. Fenner, A.F. El-Kattan. Targeting intestinal transporters for optimizing oral drug absorption. Curr Drug Metab 11 (2010) 730-742.

[13] J.D. Schuetz, P.W. Swaan, D.J. Tweedie. The role of transporters in toxicity and disease. Drug Metab Dispos 42 (2014) 541-545. 
[14] T.D. Bjornsson, J.T. Callaghan, H.J. Einolf, V. Fischer, L. Gan, S. Grimm, J. Kao, S.P. King, G. Miwa, L. $\mathrm{Ni}$, G. Kumar, J. McLeod, R.S. Obach, S. Roberts, A. Roe, A. Shah, F. Snikeris, J.T. Sullivan, D. Tweedie, J.M. Vega, J. Walsh, S.A. Wrighton, R. Pharmaceutical, G. Manufacturers of America Drug Metabolism/Clinical Pharmacology Technical Working, F.D.A.C.f.D. Evaluation, Research. The conduct of in vitro and in vivo drug-drug interaction studies: a Pharmaceutical Research and Manufacturers of America (PhRMA) perspective. Drug Metab Dispos 31 (2003) 815-832.

[15] X. Qiu, H. Zhang, Y. Lai. Quantitative targeted proteomics for membrane transporter proteins: method and application. AAPS J 16 (2014) 714-726.

[16] L.G. Zhang D., Ding X., Lu C. Preclinical experimental models of drug metabolism and disposition in drug discovery and development. Acta Pharmaceutica Sinica B, 2012, pp. 549-561.

[17] K. Horie, F. Tang, R.T. Borchardt. Isolation and characterization of Caco-2 subclones expressing high levels of multidrug resistance protein efflux transporter. Pharm Res 20 (2003) 161-168.

[18] M.D. Troutman, D.R. Thakker. Novel experimental parameters to quantify the modulation of absorptive and secretory transport of compounds by P-glycoprotein in cell culture models of intestinal epithelium. Pharm Res 20 (2003) 1210-1224.

[19] A. Balakrishnan, N. Hussainzada, P. Gonzalez, M. Bermejo, P.W. Swaan, J.E. Polli. Bias in estimation of transporter kinetic parameters from overexpression systems: Interplay of transporter expression level and substrate affinity. J Pharmacol Exp Ther 320 (2007) 133-144.

[20] Y. Shirasaka, T. Sakane, S. Yamashita. Effect of P-glycoprotein expression levels on the concentration-dependent permeability of drugs to the cell membrane. J Pharm Sci 97 (2008) 553565.

[21] K.R. Korzekwa, S. Nagar, J. Tucker, E.A. Weiskircher, S. Bhoopathy, I.J. Hidalgo. Models to predict unbound intracellular drug concentrations in the presence of transporters. Drug Metab Dispos $\mathbf{4 0}$ (2012) 865-876.

[22] D.A. Smith, L. Di, E.H. Kerns. The effect of plasma protein binding on in vivo efficacy: misconceptions in drug discovery. Nat Rev Drug Discov 9 (2010) 929-939.

[23] T. Yoshikado, K. Toshimoto, T. Nakada, K. Ikejiri, H. Kusuhara, K. Maeda, Y. Sugiyama. Comparison of Methods for Estimating Unbound Intracellular-to-Medium Concentration Ratios in Rat and Human Hepatocytes Using Statins. Drug Metab Dispos 45 (2017) 779-789.

[24] M.K. DeGorter, R.B. Kim. Use of transgenic and knockout mouse models to assess solute carrier transporter function. Clin Pharmacol Ther 89 (2011) 612-616.

[25] A.H. Schinkel, J.J. Smit, O. van Tellingen, J.H. Beijnen, E. Wagenaar, L. van Deemter, C.A. Mol, M.A. van der Valk, E.C. Robanus-Maandag, H.P. te Riele, et al. Disruption of the mouse mdr1a Pglycoprotein gene leads to a deficiency in the blood-brain barrier and to increased sensitivity to drugs. Cell 77 (1994) 491-502.

[26] C.B. Washington, H.R. Wiltshire, M. Man, T. Moy, S.R. Harris, E. Worth, P. Weigl, Z. Liang, D. Hall, L. Marriott, T.F. Blaschke. The disposition of saquinavir in normal and P-glycoprotein deficient mice, rats, and in cultured cells. Drug Metab Dispos 28 (2000) 1058-1062.

[27] A.H. Schinkel, U. Mayer, E. Wagenaar, C.A. Mol, L. van Deemter, J.J. Smit, M.A. van der Valk, A.C. Voordouw, H. Spits, O. van Tellingen, J.M. Zijlmans, W.E. Fibbe, P. Borst. Normal viability and altered pharmacokinetics in mice lacking mdr1-type (drug-transporting) P-glycoproteins. Proc Natl Acad Sci U S A 94 (1997) 4028-4033.

[28] J.W. Polli, J.L. Jarrett, S.D. Studenberg, J.E. Humphreys, S.W. Dennis, K.R. Brouwer, J.L. Woolley. Role of P-glycoprotein on the CNS disposition of amprenavir (141W94), an HIV protease inhibitor. Pharm Res 16 (1999) 1206-1212.

[29] Y. Zhang, C. Bachmeier, D.W. Miller. In vitro and in vivo models for assessing drug efflux transporter activity. Adv Drug Deliv Rev 55 (2003) 31-51.

[30] M. Yamazaki, W.E. Neway, T. Ohe, I. Chen, J.F. Rowe, J.H. Hochman, M. Chiba, J.H. Lin. In vitro substrate identification studies for p-glycoprotein-mediated transport: species difference and predictability of in vivo results. J Pharmacol Exp Ther 296 (2001) 723-735. 
[31] J.B. Houston, A. Galetin. Methods for predicting in vivo pharmacokinetics using data from in vitro assays. Curr Drug Metab 9 (2008) 940-951.

[32] J.O. Miners, P.I. Mackenzie, K.M. Knights. The prediction of drug-glucuronidation parameters in humans: UDP-glucuronosyltransferase enzyme-selective substrate and inhibitor probes for reaction phenotyping and in vitro-in vivo extrapolation of drug clearance and drug-drug interaction potential. Drug Metab Rev 42 (2010) 196-208.

[33] H.M. Jones, H.A. Barton, Y. Lai, Y.A. Bi, E. Kimoto, S. Kempshall, S.C. Tate, A. El-Kattan, J.B. Houston, A. Galetin, K.S. Fenner. Mechanistic pharmacokinetic modeling for the prediction of transportermediated disposition in humans from sandwich culture human hepatocyte data. Drug Metab Dispos 40 (2012) 1007-1017.

[34] M.J. Zamek-Gliszczynski, C.A. Lee, A. Poirier, J. Bentz, X. Chu, H. Ellens, T. Ishikawa, M. Jamei, J.C. Kalvass, S. Nagar, K.S. Pang, K. Korzekwa, P.W. Swaan, M.E. Taub, P. Zhao, A. Galetin, C. International Transporter. ITC recommendations for transporter kinetic parameter estimation and translational modeling of transport-mediated PK and DDIs in humans. Clin Pharmacol Ther 94 (2013) 64-79.

[35] S. Bosgra, E. van de Steeg, M.L. Vlaming, K.C. Verhoeckx, M.T. Huisman, M. Verwei, H.M. Wortelboer. Predicting carrier-mediated hepatic disposition of rosuvastatin in man by scaling from individual transfected cell-lines in vitro using absolute transporter protein quantification and PBPK modeling. Eur J Pharm Sci 65 (2014) 156-166.

[36] S. Neuhoff, K.R. Yeo, Z. Barter, M. Jamei, D.B. Turner, A. Rostami-Hodjegan. Application of permeability-limited physiologically-based pharmacokinetic models: part II - prediction of Pglycoprotein mediated drug-drug interactions with digoxin. J Pharm Sci 102 (2013) 3161-3173.

[37] A. Rostami-Hodjegan, G.T. Tucker. Simulation and prediction of in vivo drug metabolism in human populations from in vitro data. Nat Rev Drug Discov 6 (2007) 140-148.

[38] S. Neuhoff, K.R. Yeo, Z. Barter, M. Jamei, D.B. Turner, A. Rostami-Hodjegan. Application of permeability-limited physiologically-based pharmacokinetic models: part I-digoxin pharmacokinetics incorporating P-glycoprotein-mediated efflux. J Pharm Sci 102 (2013) 3145-3160.

[39] J.M. Dietschy. Mechanisms for the intestinal absorption of bile acids. J Lipid Res 9 (1968) 297-309.

[40] S.N. Marcus, C.D. Schteingart, M.L. Marquez, A.F. Hofmann, Y. Xia, J.H. Steinbach, H.T. Ton-Nu, J. Lillienau, M.A. Angellotti, A. Schmassmann. Active absorption of conjugated bile acids in vivo. Kinetic parameters and molecular specificity of the ileal transport system in the rat. Gastroenterology 100 (1991) 212-221.

[41] P.A. Dawson, T. Lan, A. Rao. Bile acid transporters. J Lipid Res 50 (2009) 2340-2357.

[42] R.E. Morgan, M. Trauner, C.J. van Staden, P.H. Lee, B. Ramachandran, M. Eschenberg, C.A. Afshari, C.W. Qualls, Jr., R. Lightfoot-Dunn, H.K. Hamadeh. Interference with bile salt export pump function is a susceptibility factor for human liver injury in drug development. Toxicol Sci 118 (2010) 485-500.

[43] S. Dawson, S. Stahl, N. Paul, J. Barber, J.G. Kenna. In vitro inhibition of the bile salt export pump correlates with risk of cholestatic drug-induced liver injury in humans. Drug Metab Dispos $\mathbf{4 0}$ (2012) 130-138.

[44] K. Kock, B.C. Ferslew, I. Netterberg, K. Yang, T.J. Urban, P.W. Swaan, P.W. Stewart, K.L. Brouwer. Risk factors for development of cholestatic drug-induced liver injury: inhibition of hepatic basolateral bile acid transporters multidrug resistance-associated proteins 3 and 4. Drug Metab Dispos 42 (2014) 665-674.

[45] J.M. Pedersen, P. Matsson, C.A. Bergstrom, J. Hoogstraate, A. Noren, E.L. LeCluyse, P. Artursson. Early identification of clinically relevant drug interactions with the human bile salt export pump (BSEP/ABCB11). Toxicol Sci 136 (2013) 328-343.

[46] K. Yang, K. Kock, A. Sedykh, A. Tropsha, K.L. Brouwer. An updated review on drug-induced cholestasis: mechanisms and investigation of physicochemical properties and pharmacokinetic parameters. J Pharm Sci 102 (2013) 3037-3057. 
[47] A.D. Rodrigues, Y. Lai, M.E. Cvijic, L.L. Elkin, T. Zvyaga, M.G. Soars. Drug-induced perturbations of the bile acid pool, cholestasis, and hepatotoxicity: mechanistic considerations beyond the direct inhibition of the bile salt export pump. Drug Metab Dispos 42 (2014) 566-574.

[48] P.B. Watkins. Idiosyncratic liver injury: challenges and approaches. Toxicol Pathol 33 (2005) 1-5.

[49] E.M. Leslie, P.B. Watkins, R.B. Kim, K.L. Brouwer. Differential inhibition of rat and human Na+dependent taurocholate cotransporting polypeptide (NTCP/SLC10A1)by bosentan: a mechanism for species differences in hepatotoxicity. J Pharmacol Exp Ther 321 (2007) 1170-1178.

[50] J.L. Woodhead, K. Yang, S.Q. Siler, P.B. Watkins, K.L. Brouwer, H.A. Barton, B.A. Howell. Exploring BSEP inhibition-mediated toxicity with a mechanistic model of drug-induced liver injury. Front Pharmacol 5 (2014) 240.

[51] K. Yang, J.L. Woodhead, P.B. Watkins, B.A. Howell, K.L. Brouwer. Systems pharmacology modeling predicts delayed presentation and species differences in bile acid-mediated troglitazone hepatotoxicity. Clin Pharmacol Ther 96 (2014) 589-598.

[52] X. Cao, L.X. Yu, C. Barbaciru, C.P. Landowski, H.C. Shin, S. Gibbs, H.A. Miller, G.L. Amidon, D. Sun. Permeability dominates in vivo intestinal absorption of P-gp substrate with high solubility and high permeability. Mol Pharm 2 (2005) 329-340.

[53] C.L. Cummins, L. Salphati, M.J. Reid, L.Z. Benet. In vivo modulation of intestinal CYP3A metabolism by P-glycoprotein: studies using the rat single-pass intestinal perfusion model. J Pharmacol Exp Ther 305 (2003) 306-314.

[54] K.S. Pang. Modeling of intestinal drug absorption: roles of transporters and metabolic enzymes (for the Gillette Review Series). Drug Metab Dispos 31 (2003) 1507-1519.

[55] M. Jamei, D. Turner, J. Yang, S. Neuhoff, S. Polak, A. Rostami-Hodjegan, G. Tucker. Population-based mechanistic prediction of oral drug absorption. AAPS J 11 (2009) 225-237.

[56] A.S. Darwich, S. Neuhoff, M. Jamei, A. Rostami-Hodjegan. Interplay of metabolism and transport in determining oral drug absorption and gut wall metabolism: a simulation assessment using the "Advanced Dissolution, Absorption, Metabolism (ADAM)" model. Curr Drug Metab 11 (2010) 716729.

[57] A. Bruyere, X. Decleves, F. Bouzom, K. Ball, C. Marques, X. Treton, M. Pocard, P. Valleur, Y. Bouhnik, Y. Panis, J.M. Scherrmann, S. Mouly. Effect of variations in the amounts of P-glycoprotein (ABCB1), BCRP (ABCG2) and CYP3A4 along the human small intestine on PBPK models for predicting intestinal first pass. Mol Pharm 7 (2010) 1596-1607.

[58] A. Olivares-Morales, A. Ghosh, L. Aarons, A. Rostami-Hodjegan. Development of a Novel Simplified PBPK Absorption Model to Explain the Higher Relative Bioavailability of the OROS(R) Formulation of Oxybutynin. AAPS J 18 (2016) 1532-1549.

[59] M.S. Alavijeh, M. Chishty, M.Z. Qaiser, A.M. Palmer. Drug metabolism and pharmacokinetics, the blood-brain barrier, and central nervous system drug discovery. NeuroRx 2 (2005) 554-571.

[60] M. Feher, E. Sourial, J.M. Schmidt. A simple model for the prediction of blood-brain partitioning. Int J Pharm 201 (2000) 239-247.

[61] S. Vilar, M. Chakrabarti, S. Costanzi. Prediction of passive blood-brain partitioning: straightforward and effective classification models based on in silico derived physicochemical descriptors. $J \mathrm{Mol}$ Graph Model 28 (2010) 899-903.

[62] J.A. Platts, M.H. Abraham, Y.H. Zhao, A. Hersey, L. ljaz, D. Butina. Correlation and prediction of a large blood-brain distribution data set--an LFER study. Eur J Med Chem 36 (2001) 719-730.

[63] N.J. Abbott, A.A. Patabendige, D.E. Dolman, S.R. Yusof, D.J. Begley. Structure and function of the blood-brain barrier. Neurobiol Dis 37 (2010) 13-25.

[64] Y. Yamamoto, P.A. Valitalo, D.J. van den Berg, R. Hartman, W. van den Brink, Y.C. Wong, D.R. Huntjens, J.H. Proost, A. Vermeulen, W. Krauwinkel, S. Bakshi, V. Aranzana-Climent, S. Marchand, C. Dahyot-Fizelier, W. Couet, M. Danhof, J.G. van Hasselt, E.C. de Lange. A Generic MultiCompartmental CNS Distribution Model Structure for 9 Drugs Allows Prediction of Human Brain Target Site Concentrations. Pharm Res 34 (2017) 333-351. 
[65] A.M. Palmer, M.S. Alavijeh. Translational CNS medicines research. Drug Discov Today 17 (2012) 1068-1078.

[66] A.K. Deo, F.P. Theil, J.M. Nicolas. Confounding parameters in preclinical assessment of blood-brain barrier permeation: an overview with emphasis on species differences and effect of disease states. Mol Pharm 10 (2013) 1581-1595.

[67] F. Fenneteau, P. Poulin, F. Nekka. Physiologically based predictions of the impact of inhibition of intestinal and hepatic metabolism on human pharmacokinetics of CYP3A substrates. J Pharm Sci 99 (2010) 486-514.

[68] K. Ball, F. Bouzom, J.M. Scherrmann, B. Walther, X. Decleves. Physiologically based pharmacokinetic modelling of drug penetration across the blood-brain barrier--towards a mechanistic IVIVE-based approach. AAPS J 15 (2013) 913-932.

[69] K. Ball, F. Bouzom, J.M. Scherrmann, B. Walther, X. Decleves. Development of a physiologically based pharmacokinetic model for the rat central nervous system and determination of an in vitro-in vivo scaling methodology for the blood-brain barrier permeability of two transporter substrates, morphine and oxycodone. J Pharm Sci 101 (2012) 4277-4292.

[70] N. Notkina, C. Dahyot-Fizelier, A.K. Gupta. In vivo microdialysis in pharmacological studies of antibacterial agents in the brain. Br J Anaesth 109 (2012) 155-160.

[71] W.C.-Y. Benet LZ, Hebert MF, Wacher VJ. Intestinal drug metabolism and antitransport processes: a potential paradigm shift in oral drug delivery. Journal of Controlled Release 2 (1996) 139-143.

[72] V.J. Wacher, L. Salphati, L.Z. Benet. Active secretion and enterocytic drug metabolism barriers to drug absorption. Adv Drug Deliv Rev 46 (2001) 89-102.

[73] Y.Y. Lau, Y. Huang, L. Frassetto, L.Z. Benet. effect of OATP1B transporter inhibition on the pharmacokinetics of atorvastatin in healthy volunteers. Clin Pharmacol Ther 81 (2007) 194-204.

[74] J.A. Grillo, P. Zhao, J. Bullock, B.P. Booth, M. Lu, K. Robie-Suh, E.G. Berglund, K.S. Pang, A. Rahman, L. Zhang, L.J. Lesko, S.M. Huang. Utility of a physiologically-based pharmacokinetic (PBPK) modeling approach to quantitatively predict a complex drug-drug-disease interaction scenario for rivaroxaban during the drug review process: implications for clinical practice. Biopharm Drug Dispos 33 (2012) 99-110. 